\begin{tabular}{|c|l|}
\hline Title & End-Crosslinking Gelation of Poly(amide acid) Gels studied with Scanning Microscopic Light Scattering \\
\hline Author(s) & Furukawa, Hidemitsu; Kobay ashi, Mizuha; Miy ashita, Y oshiharu; Horie, Kazuy uki \\
\hline Citation & $\begin{array}{l}\text { High Performance Polymers, 18(5), 837-847 } \\
\text { https://doi.org/10.1177/0954008306068270 }\end{array}$ \\
\hline Issue Date & 2006-10 \\
\hline Doc URL & http://hdl.handle.net/2115/14926 \\
\hline Rights & Copyright $\odot$ 2006 SA GE Publications HIGH PERFORMA NCE POLY MERS \\
\hline Type & article (author version) \\
\hline File Information & HPP18 5.pdf \\
\hline
\end{tabular}

Instructions for use 


\title{
End-Crosslinking Gelation of Poly(amide acid) Gels studied with Scanning Microscopic Light Scattering
}

\author{
HIDEMITSU FURUKAWA
}

Division of Biological Sciences, Graduate School of Science, Hokkaido University, N10, W8, Kita-ku, Sapporo-shi 060-0810, Japan

\section{MIZUHA KOBAYASHI}

Department of Organic and Polymer Materials Chemistry, Tokyo University of Agriculture and Technology, 2-24-16 Naka-cho, Koganei-shi, Tokyo 184-8588, Japan

\section{YOSHIHARU MIYASHITA}

Department of Chemistry and Material Engineering, Ibaraki National College of Technology, 866 Nakane, Hitachinaka-shi, Ibaraki 312-8508, Japan

\section{KAZUYUKI HORIE ${ }^{\dagger}$}

Department of Organic and Polymer Materials Chemistry, Tokyo University of Agriculture and Technology, 2-24-16 Naka-cho, Koganei-shi, Tokyo 184-8588, Japan

(Received 6 February 2006; accepted 28 February 2006)

Abstract: Network formation in gelation process of end-crosslinked poly(amide acid) gels, which are the precursor of end-crosslinked polyimide gels, were studied by scanning dynamic light scattering. This gelation process is essentially non-reversible due to the formation of covalent bonds. The molecular structure formed in gelation process is controlled by varying the equivalence ratio of end-crosslinker to oligomer in preparation. It is found that a couple of relaxation modes are observed in gelation point

\footnotetext{
+ Present Address: Industrial Application Division, Japan Synchrotron Radiation Research Institute (SPring-8), 1-1-1, Kouto, Sayo, Hyogo 679-5198, Japan
} 
even for semi-rigid poly(amide acid) solutions. It is similar to flexible polymer solutions and convenient to characterize the molecular structure in gelation processes. The fast mode is related to the cooperative diffusion process of network structure formed by overlapped macromolecules, i.e., gel mode, which determines the averaged mesh-size of the network structure. The slow mode is related to the relaxation time of the formation of the transient network structure, which shows slowing down close to gelation point and disappears after gelation due to the formation of permanent network structure.

Key Words: relaxation time, gel mode, semi-dilute solution, diffusion coefficient, percolation

\section{INTRODUCTION}

Recently, we have proposed a novel preparation approach for high-performance end-crosslinked poly(amide acid) and polyimide gels with rigid main chains by using polycondensation. ${ }^{1}$ In the previous studies, ${ }^{1,2}$ we found that the physical properties of the polyimide gels, such as elastic moduli, solubility and transparency, can be controlled by the changes in the rigidity and the solubility of main chains, and the number of repeating units of oligomer sub-chains. By using this end-crosslinking preparation method, we thought that a kind of ideal jungle-gym network structure of polyimide could be synthesized. In the present study, in order to clarify whether such a network structure is formed or not, a chemically end-linking gelation of poly(amide acid) gels, which are the precursor of end-crosslinked polyimide gels, has been studied by using scanning microscopic light scattering (SMILS). ${ }^{3,4}$ By virtue of SMILS, ensemble-averaged dynamic structure factor of poly(amide acid) gels is determined rigorously and the molecular structure inside the gels is precisely characterized by analyzing the structure factor. The present study is, of course, only the first step of the analysis of the end-crosslinking gelation process in poly(amide acid) gels. However, if once the analysis scheme is established, this scheme can be applied for the characterization of many kinds of transparent aromatic rigid polymer gels prepared in organic solvents, in order to know many properties of the network structure in the gels, such as, mesh-size, defects in network, concentration fluctuation, i.e., inhomogenities, 
and so on. Especially, by using such an analysis scheme, we can find the suitable preparing condition of the homogeneous network structure in polymer gels.

By the way, the light scattering studies of chemical gelation process in polymeric systems ${ }^{5}$ induced by chemical crosslinking forming covalent bonds have been little performed comparing to physical gelation. ${ }^{6}$ Because the mechanism of the chemical gelation is much more complex than physical gelation, many unsolved problems remain especially concerning inhomogeneities, ${ }^{7}$ such as microsyneresis, speckle pattern, non-ergodicity, and so on. The end-crosslinking system of poly(amide acid) may become one of appropriate systems to elucidate the unsolved problems about the chemical gelation. Some DLS studies of the chemical gelation of vinylpolymer systems have been performed, e.g., in $\operatorname{poly}\left(N\right.$-isopropylacrylamide) ${ }^{8}$ poly(methyl methacrylate), ${ }^{9,10}$ poly(dimethylsiloxane) gels, ${ }^{11}$ and other randomly branched polymer systems. ${ }^{12}$ For these polymer systems, however, it is generally difficult to observe the chemical gelation because the process of the chemical reaction progresses during the observation. Moreover, these gels are formed with flexible main chains, so that both their coiled structures and the entanglement among the flexible chains prevent from observing the network structure. On the other hand, the semi-rigid main chains in poly(amide acid) gels will form well-swollen and not-entangled network structure due to the rigidity of aromatic poly(amide acid) and the small length of oligomeric chains. Further, in the present system, the approach to a gelation point can be appropriately controlled by varying the equivalence ratio of end-crosslinker to oligomer. It suggests that end-crosslinked poly(amide acid) gels are convenient and suitable for the systematic study of the chemical gelation process. This point also should be clarified in the present study.

\section{EXPERIMENTAL}

Scheme 1 shows a preparation method of the pre-gel solutions and sample gels. Both monomers and an end-crosslinker, 4,4'(hexafluoroisopropylidene)diphthalic anhydride (6FDA), 4,4'-oxydianiline (ODA), and 1,3,5-tris(4-aminophenyl)benzene ${ }^{1}$ (TAPB), were dissolved in $N, N$-dimethylformamide (DMF) at room temperature separately. All DMF solutions were filtered with $0.2 \mu \mathrm{m}$ membrane filter for optical refinement before mixing. The ODA/DMF solution was slowly dropped onto the 6FDA/DMF solution to prepare a $24 \mathrm{wt} \%$ oligo(amide acid) solution, in which the molar 
ratio of 6FDA/ODA was fixed to be 2/1. The molecular weight and its distribution of the oligomer was measured to be $M_{\mathrm{n}}=1.8 \times 10^{3}$ (scaled in terms of polystyrene) and $M_{\mathrm{w}} / M_{\mathrm{n}}=1.1$ by gel permeation chromatography. Then the oligomer/DMF solution was diluted and onto this solution TAPB/DMF solution was slowly dropped with varying the equivalence ratios of end-crosslinker TAPB to oligomer, $R_{v}$, which can be expressed as $R_{v}=3[\mathrm{TAPB}] / 2[$ oligomer] with molar concentrations. Firstly, in order to choose a proper polymer concentration for gel formation, the solutions were prepared for a fixed value of $R_{v} \cong 1$ with $4,8,10,16$, and $24 \mathrm{wt} \%$ polymer concentrations. Then 10,16 , and $24 \mathrm{wt} \%$ solutions gelled in a few minutes, while 4 or $8 \mathrm{wt} \%$ solution did not form a gel. All the solutions kept transparent after gelation. Thus $10 \mathrm{wt} \%$ was chosen to be the nearest to the critical gelation point. Next $10 \mathrm{wt} \%$ solutions were prepared for various values of $R_{v}$ as $0,0.30,0.61,0.95$, and 2.40 . A previous theory ${ }^{13}$ predicts that the end-linking gelation occurs in the range of $(1 / \sqrt{2}) \leq R_{v} \leq \sqrt{2}$. Thus we expected that the solution will become gelled only for $R_{v} \cong 0.95$ and the other solutions will not be gelled.

The scanning DLS measurements have been preformed at $30.0^{\circ} \mathrm{C}$ in the scattering angle range of $\theta=30^{\circ} \sim 125^{\circ}$ with a scanning light scattering apparatus. ${ }^{3,4}$ With this apparatus, many measurements can be successively performed at different positions in a sample. Hence, we can obtain a time- and space-averaged correlation function, i.e., an ensemble-averaged correlation function to overcome the non-ergodicity of inhomogeneous media. ${ }^{14}$ The photon detection was carried out with a homodyne technique. For each sample, the time-averaged homodyne correlation functions $g_{\mathrm{t}}^{(2)}(\mathbf{q}, \tau)$ were determined at over a hundred points, where $\mathbf{q}$ is scattering vector and $\tau$ is correlation time. Then the ensemble-averaged heterodyne correlation function $g_{\mathrm{en}}^{(1)}(\mathbf{q}, \tau)$ was calculated by using the following equation ${ }^{14}$

$$
g_{\mathrm{en}}^{(1)}(\mathbf{q}, \tau)=\left\langle\langle I\rangle_{\mathrm{t}} \gamma^{-1} \sqrt{1+g_{\mathrm{t}}^{(2)}(\mathbf{q}, \tau)-g_{\mathrm{t}}^{(2)}(\mathbf{q}, 0)}\right\rangle_{\mathrm{sp}} /\langle I\rangle_{\mathrm{en}} .
$$

where $I$ is the scattering intensity and $\gamma$ is an apparatus constant. In the present work, since we are interested mainly in the dynamics of fluctuation in the chemical gelation, we calculated the dynamic components of $g_{\mathrm{en}}^{(1)}(\mathbf{q}, \tau)$ as 


$$
\begin{aligned}
\Delta g_{\mathrm{en}}^{(1)}(\mathbf{q}, \tau) & =\frac{g_{\mathrm{en}}^{(1)}(\mathbf{q}, \tau)-g_{\mathrm{en}}^{(1)}(\mathbf{q}, \infty)}{g_{\mathrm{en}}^{(1)}(\mathbf{q}, 0)-g_{\mathrm{en}}^{(1)}(\mathbf{q}, \infty)} \\
& =\frac{\left\langle\langle I\rangle_{\mathrm{t}} \sqrt{1+g_{\mathrm{t}}^{(2)}(\mathbf{q}, \tau)-g_{\mathrm{t}}^{(2)}(\mathbf{q}, 0)}\right\rangle_{\mathrm{sp}}-\left\langle\langle I\rangle_{\mathrm{t}} \sqrt{2-g_{\mathrm{t}}^{(2)}(\mathbf{q}, 0)}\right\rangle_{\mathrm{sp}}}{\langle I\rangle_{\mathrm{en}}-\left\langle\langle I\rangle_{\mathrm{t}} \sqrt{2-g_{\mathrm{t}}^{(2)}(\mathbf{q}, 0)}\right\rangle_{\mathrm{sp}}} .
\end{aligned}
$$

It is convenient to use this equation, since one has no need to consider the apparatus constant $\gamma$.

To analyze $\Delta g_{\text {en }}^{(1)}(\mathbf{q}, \tau)$, a relaxation time distribution $P_{\text {en }}\left(\mathbf{q}, \tau_{\mathrm{R}}\right)$ was calculated numerically with inverse Laplace transform. In general, a monotonically decreasing correlation function can be expressed by the superposition of exponential functions as ${ }^{3,15}$

$$
\Delta g_{\mathrm{en}}^{(1)}(\tau)=N \sum_{i=1}^{n} P_{\mathrm{en}}\left(\tau_{\mathrm{R}, i}\right) \exp \left(-\tau / \tau_{\mathrm{R}, i}\right)
$$

where $\tau_{\mathrm{R}, i}=\tau_{\mathrm{R}, \min } \times\left(\tau_{\mathrm{R}, \max } / \tau_{\mathrm{R}, \min }\right)^{(i-1) / n}(i=1,2, \cdots, n)$ and $N$ is a normalized factor defined as $N=(1 / n) \log \left(\tau_{\mathrm{R}, \max } / \tau_{\mathrm{R}, \min }\right)$. Thus the nonlinear fitting with eq. (3) to $\Delta g_{\mathrm{en}}^{(1)}(\mathbf{q}, \tau)$ was performed where the analytical condition was set as $\log \left(\tau_{\mathrm{R}, \min } / \mathrm{s}\right)=-5.1, \log \left(\tau_{\mathrm{R}, \max } / \mathrm{s}\right)=1.4$, and $n=65$.

\section{RESULTS AND DISCUSSION}

Figure 1 shows the change in ensemble-averaged correlation function $\Delta g_{\text {en }}^{(1)}(\mathbf{q}, \tau)$ depending on the composition of pre-gel solution in preparation. Here, $\Delta g_{\mathrm{en}}^{(1)}(\mathbf{q}, \tau)$ is directly related to the dynamic structure factor of polymer solution, which provides information about the relaxation process induced by thermal fluctuation in polymer gels. There are two different gelation processes, as shown in figure 1. Gelation I is a process from $R_{v}=0$ to a gelation point, and Gelation II is another process from $R_{v}=\infty$ to another gelation point. As shown in figure $1, \Delta g_{\mathrm{en}}^{(1)}(\mathbf{q}, \tau)$ shows two relaxation modes except $R_{v}=0.95$. A fast mode of relaxation is observed around $\tau=10^{-6} \sim 10^{-4} \mathrm{~s}$, and the shoulder position of the fast mode is almost independent of $R_{v}$. On the other hand, a slow mode of relaxation is observed in $\tau \geq 10^{-2} \mathrm{~s}$ except for $R_{v}=0.95$. The peak position of the slow mode strongly depends on $R_{v}$ and its 
relaxation slows down as $R_{v}$ approaches to the gelation points in both Gelation I and II processes.

Figure 2 shows the relaxation-time distribution $P_{\mathrm{en}}\left(\mathbf{q}, \tau_{\mathrm{R}}\right)$, which is calculated from $\Delta g_{\text {en }}^{(1)}(\mathbf{q}, \tau)$. There exist both fast and slow modes obviously except for $R_{v}=0.95$. In Gelation I process, as $R_{v}$ increases toward to the gelation point in Gelation I prosess, the slow mode becomes slower, while the fast mode remains at the same relaxation time. However, the distribution width of the fast mode alters dramatically close to the gelation point. The relaxation time distribution of $R_{v}=2.4$ in Gelation II process is similar to $R_{v}=0$ in Gelation I process.

In the cases of semi-dilute solutions of flexible polymer chains, both the similar fast and slow modes are usually observed with DLS. ${ }^{16}$ In the semi-dilute solutions, where the concentration of polymer is above the overlap concentration, polymer chain interpenetrates each other and makes transient network structure, even when they have no interaction among each other. For those cases, the fast mode is assigned to the cooperative diffusion of the transient network structure, i.e., gel mode, and the slow mode corresponds to the relaxation process of the transient network. In the present study for semi-rigid, short oligomer chains, both fast and slow modes are also observed. Even in this case, the fast mode also corresponds to gel mode due to the cooperative diffusion of transient or permanent network structure. The slow mode is correlated to the relaxation time of the transient network structure formed by overlapped macromolecules. Based on these points of view, the behavior observed in figure 2 is explained as follows.

At $R_{v}=0$, both fast and slow modes are observed, while no reaction is made in this pre-gel solution. We think rigid oligomer in this solution already overlapped each other due to its extended structure. So that the solution behaves as the semi-dilute polymer solution, and both the gel mode and the relaxation of transient network structure are observed. At $R_{v}=0.30$ and 0.61 , the distribution of the gel mode becomes broad and the slow mode slows down as $R_{v}$ increases. Below the gelation point, the increase of $R_{v}$ causes the formation of various-size clusters of sol components, which are made of end-crosslinked oligomers, and there are various mesh sizes inside the clusters and among the overlapped clusters. Especially, the large cluster affects on the relaxation time. So that the relaxation time distribution of the gel mode becomes broad and the characteristic relaxation time of the slow mode becomes very 
large. At $R_{v}=0.95$, the profile of the distribution function changes dramatically. Above the gelation point, the infinite size cluster is formed and the network structure becomes to be fixed in the gel solution. So that the profile of the gel mode becomes very sharp and the slow relaxation of the transient network structure diminishes completely. The diminishment of the slow mode for $R_{v}=0.95$ means that the self-diffusion of the clusters is suppressed by the chemical gelation by end-crosslinking and the formation of infinite network. Thus the diminishment of the slow mode is convenient to distinguish gelled solution from sol solution.

On the other hand, $R_{v}=2.4$ belongs to the Gelation II process and there are 2.4 times larger amount of the end-closslinker than oligomers. So that almost all of oligomer is end-capped by a large amount of end-crosslinker and any cluster is hardly formed. Thus the profile of relaxation-time distribution at $R_{v}=2.4$ is very similar to that of $R_{v}=0$,

For semi-dilute solutions of usual flexible polymer, the relaxation time of the fast gel-mode depends on the polymer concentration according to the blob model. ${ }^{16}$ In the present study, the poly(amide acid) is not as flexible as usual vinylpolymer. Does the rigidity of poly(amide acid) effect on the gel mode? In results, when the polymer concentration is fixed at $10 \mathrm{wt} \%$, the relaxation time of the gel mode is independent of $R_{v}$. It means that the rigidity and end-crosslinking does not strongly prevent the cooperative diffusion of the blobs. There may be not completely end-crosslinked jungle-gym-like network structure shown in Scheme 1 even at $R_{v}=0.95$, where many uncrosslinked ends still exist. Thus the transient or end-crosslinked network has flexible properties although the main chain of the poly(amide acid) is not flexible. However, for $R_{v}=0.30 \sim 0.61$ the relaxation time distribution of the gel mode broadens. It suggests the presence of various sizes of cluster has any effect on the distribution of the relaxation mode.

Figure 3 shows the $\log -\log$ plot of $q^{2} \tau$-scaled $\Delta g_{\text {en }}^{(1)}(\mathbf{q}, \tau)$ for the gelled sample at $R_{v}=0.95$, where $q=(4 \pi n / \lambda) \sin (\theta / 2)$ with $n=1.34$ and $\lambda=633 \mathrm{~nm}$. By $q^{2} \tau$-scaling, the profiles of the fast mode are overlapped well. It means all the relaxation is related to translational diffusion processes. The stretched exponential function, $\Delta g_{\text {en }}^{(1)}\left(q^{2} \tau\right) \cong \exp \left[-\left(q^{2} D_{\text {coop }} \tau\right)^{\beta}\right]$, was fitted numerically to $\Delta g_{\text {en }}^{(1)}\left(q^{2} \tau\right)$ of $\theta=60^{\circ}$, where $D_{\text {coop }}$ is the cooperative diffusion coefficient. By fitting we obtained 
$D_{\text {coop }}=2.2 \times 10^{-10} \mathrm{~m}^{2} / \mathrm{s}$ and $\beta=0.52$. Thus the mesh size of network is estimated as $2 \xi \cong 2.5 \mathrm{~nm}$ by using the Einstein-Stokes relationship ${ }^{16}\left[D_{\text {coop }}=k_{B} T /(6 \pi \eta \xi)\right]$.

The small value of $\beta=0.52$ suggests the existence of the slow relaxation process following just after the gel mode, which may correlate with the fractal-like structure formed during the chemical gelation, as will be discussed below. Relating to this, $P_{\text {en }}\left(\mathbf{q}, \tau_{\mathrm{R}}\right)$ at $R_{v}=0.95$ has a very small peak around $\tau_{\mathrm{R}} \cong 10^{-3} \mathrm{~s}$ in figure 2 . However, the main peak of the gel mode is somewhat sharp as shown in figure 2. Thus it suggests the nanometer-scale mesh size at $R_{v}=0.95$ is almost uniform and $2.5 \mathrm{~nm}$ is a good estimation of the averaged mesh size in poly(amide acid) gels. Moreover, the relaxation time and sharpness of the gel mode at $R_{v}=0.95$ looks similar to those at $R_{v}=0$. It means that the network structure of the end-crosslinked poly(amide acid) gels is not so different from that of the transient network of oligo(amide acid). Thus the previous structure before gelation is not disturbed so much after the end-crosslinking gelation. Thus it suggests that by using rigid oligomer and symmetric end-crosslinker, homogeneous poly(amide acid) network can be prepared. ${ }^{1}$ Of course, the homogeneity of the end-crosslinked poly(amide acid) network structure should be confirmed more rigorously in further investigations. In order to achieve it, we are studying the effect of the preparation condition of end-crosslinked poly(amide acid) gels on their network structure more systematically and it will be reported elsewhere.

In figure 3, we can see the power-law profile of $\Delta g_{\text {en }}^{(1)}\left(q^{2} \tau\right)$ in $10^{11}<q^{2} \tau<10^{13}$, which looks like a characteristic profile for sol-gel transition correspond to fractal-like structure. Martin et al. proposed a power law function with critical exponents at a gel point as ${ }^{17}$

$$
\Delta g_{e n}^{(1)}(\mathbf{q}, \tau) \sim \tau^{-\beta /(v+k)} \sim \tau^{-\alpha}
$$

where $\beta, v, k$, and $\alpha$ are the critical exponents. While the classical mean field theory yields $\beta=1$ and $v=0.5,{ }^{17}$ the percolation theory yields $\beta=0.39$ and $v=0.89 .{ }^{17}$ The exponent, ${ }^{18} k$, is predicted as $k=0.75$ by classical electrical analogy and $k=1.33$ by dynamic scaling. Thus the predicted slopes of $\alpha=0.80$ by the classical theories and $\alpha=0.18$ by the advanced theories are shown in Figure 3 for comparison. The long tail of $\Delta g_{\mathrm{en}}^{(1)}\left(q^{2} \tau\right)$ in $10^{11}<q^{2} \tau<10^{13}$ is close to $\alpha=0.18$.

However, the value of $\alpha$ cannot be determined quantitatively within the present 
study because there presumably exists some deviation from the critical point shown in figure 4. The lower gelation concentration at the critical point, $C_{g}$, probably is still lower than $10 \mathrm{wt} \%$. Thus at $10 \mathrm{wt} \%$ there are a couple of gelation points of $R_{v}$, which exist below and above 1 . Accordingly, $R_{v}=0.95$ is an intermediate point located between both the gelation points. Ideally, the power-law profile at the critical point should be observed at $C_{g}$ and $R_{v}=1$. Such an observation will illuminate the critical phenomena of the chemical gelation. On the other hand, the present estimation of the averaged mesh-size at $R_{v}=0.95$, which is $2.5 \mathrm{~nm}$, is related to the polymer concentration of $10 \mathrm{wt} \%$. As discussed above, the behavior of the present poly(amide acid) solution is similar to the semi-dilute solution of flexible polymer. It suggests that the mesh size estimated from gel mode depends on the polymer concentration. The rigidity has no strong effect on the nanoscale network structure.

From a different point of view, the present polymer concentration is so close to the gelation point and it is also close to the overlap concentration. It suggest that the observed mesh size by the fast gel-mode corresponds to the molecular dimension of oligo(amide acid). Here, the end-to-end distance of the oligo(amide acid) is numerically calculated as $2.4 \mathrm{~nm}$ by simple molecular modeling software (CambridgeSoft Chem3D). This calculated end-to-end distance of oligo(amide acid) is very close to the measured mesh size actually.

Thus the chemical gelation process of poly(amide acid) solution can be well characterized by dynamic light scattering. Around the gelation point, the diminishment of the slow mode directly corresponds to the formation of infinite network structure and the fast gel-mode provides information about the mesh-size and the inhomogeneity of molecular structure. Simultaneously, the critical behavior around the critical gelation point can be also observed well. It means this poly(amide acid) solution will be suitable system for detailed studies of the chemical gelation. Further, by controlling the functionality of end-crosslinker, the rigidity and the solubility of main chains, and the number of monomer units in oligomer, it is possible to elucidate many unsolved problems in chemical gelation processes, especially, the origin of the frozen inhomogeneity in polymer gels formed in gelation process. In addition, the properties of the poly(amide acid) gels will also be improved by such a control of chemical gelation. Both rigidity and end-crosslinking of the present system are crucial advantages for application as well as elucidation of chemical gelation. 


\section{SUMMARY}

In the present study, the characteristics of sol-gel transition in end-crosslinked poly(amide acid) gels with semi-rigid main chains were studied by SMILS. It is found that the chemical gelation process can be clearly observed by varying the preparation concentration ratio of end-crosslinker to oligomer molecules. A couple of relaxation modes were observed as a function of the deviation from a gelation point. The fast mode corresponds to the cooperative diffusion in semi-dilute solution and gels. The slow mode relates to the relaxation time of transient network structure. The analysis of these modes yields the information on the molecular structure and dynamics during the chemical gelation. End-crosslinked poly(amide acid) solutions and gels with the rigid main chains behaves as such as flexible polymer solutions and gels. Thus the gelation process can be characterized by dynamic light scattering. Especially, the diminishment of the slow mode corresponds to the formation of infinite network structure. Further, the quasi-critical behavior closing up to the gelation points also can be well characterized. It means that end-crosslinked poly(amide acid) solution will be one of the key system for studies of the unsolved problems about chemical gelation. 


\section{References and Notes}

1 He J, Machida S, Kishi H, Horie K, Furukawa H, Yokota R 2002 J Polym Sci A: Polym Chem 402501

2 He J, Horie K, Yokota R, He F 2001 Polymer 424063

3 Furukawa H, Horie K, Nozaki R, Okada M 2003 Phys Rev E 68031406

4 Furukawa H, Okada M 2000 Trans Mater Res Soc Jpn 25723

$5 \quad$ Martin J E, Adolf D 1991 Ann Rev Phys Chem 42311

6 Adam M, Lairez D 1996 The Physical Properties of Polymeric Gels ed Cohen J P (London: John Wiley \& Sons Ltd) Chapter 4

7 Shibayama M 1998 Macromol Chem Phys 1991

8 Norisuye T, Takeda M, Shibayama M, 1998 Macromolecules 315316

9 Fang L, Brown W, Konak C 1991 Macromolecules 246839

10 Lesturgeon V, Nicolai T, Durand D 1999 Eur Phys J B 971

11 Adam M, Lairez D, Karpasas M, Gottlib M 1997 Macromolecules 305920

12 Trappe V, Bauer J, Weissmuller M, Burchard W 1997 Macromolecules 302365

13 Miller D R, Macosko C W 1976 Macromolecules 9206

14 Furukawa H, Hirotsu S 2002 J Phys Soc Jpn 712873

15 Ostrowsky N, Sornette D, Parker P, Pike E R 1981 Optica Acta 281059

16 Teraoka I 2002 Polymer Solutions (New York: Wiley)

17 Martin J E, Wilcoxon J P, Odinek 1991 J Phys Rev A 43858

18 Adolf D, Martin J E, Wilcoxon J P 1990 Macromolecules 23527 


\section{Scheme and figure captions}

Scheme 1. Preparation of poly(amide acid) gels end-crosslinked with symmetrical end-crosslinker of TAPB.

Figure 1. Dynamic component of ensemble-averaged autocorrelation functions $\Delta g_{e n}^{(1)}(\tau)$ at various end-crosslinker concentrations $R_{v}$. Scattering angle is $60^{\circ}$.

Figure 2. Relaxation-time distribution function $P_{\mathrm{en}}\left(\tau_{R}\right)$ at various end-crosslinker concentration $R_{v}$. Scattering angle is $40^{\circ}$. The solid curves are the logarithmic Gaussian distributions fitted to $P_{\mathrm{en}}\left(\tau_{\mathrm{R}}\right)$.

Figure 3. Profiles of $q^{2} \tau$-scaled autocorrelation function $\Delta g_{\mathrm{en}}^{(1)}\left(q^{2} \tau\right)$ near the gelation point at $R_{v}=0.95$. The broken curve is the stretched exponential function fitted to $\Delta g_{\mathrm{en}}^{(1)}\left(q^{2} \tau\right)$ of $\theta=60^{\circ}$.

Figure 4. An expected phase diagram of gelation, where the symbols indicate the prepared samples in order to choose a proper polymer concentration for gel formation $(\times)$ and the prepared samples for SMILS measurements $(\bigcirc)$. 
2

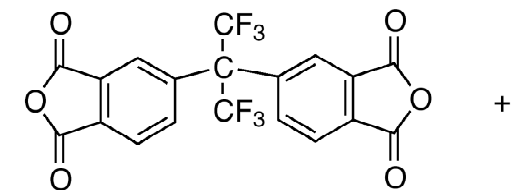

4,4'-(hexafluoroisopropylidene)diphthalic anhydride (6FDA)<smiles>Nc1ccc(Oc2ccc(N)cc2)cc1</smiles>

4,4'-oxydianiline (ODA)<smiles>CCOC(=O)c1ccc(C(F)(F)C(F)(F)F)cc1C(=O)Nc1ccc(Oc2ccc(NC(=O)c3cc(C(F)(F)C(F)(F)F)ccc3C(=O)O)cc2)cc1</smiles>

OAA(6FDA/ODA)(2/1)
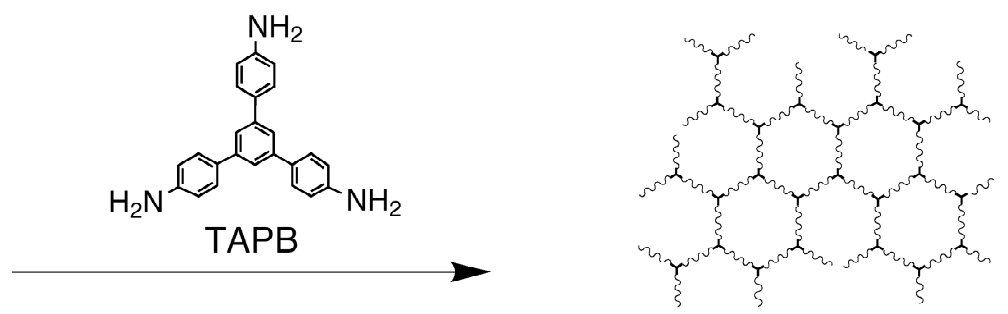

PAA(OAA(6FDA/ODA)(2/1)- v -TAPB)

Scheme 1. Preparation of poly(amide acid) gels end-crosslinked with symmetrical end-crosslinker of TAPB. 


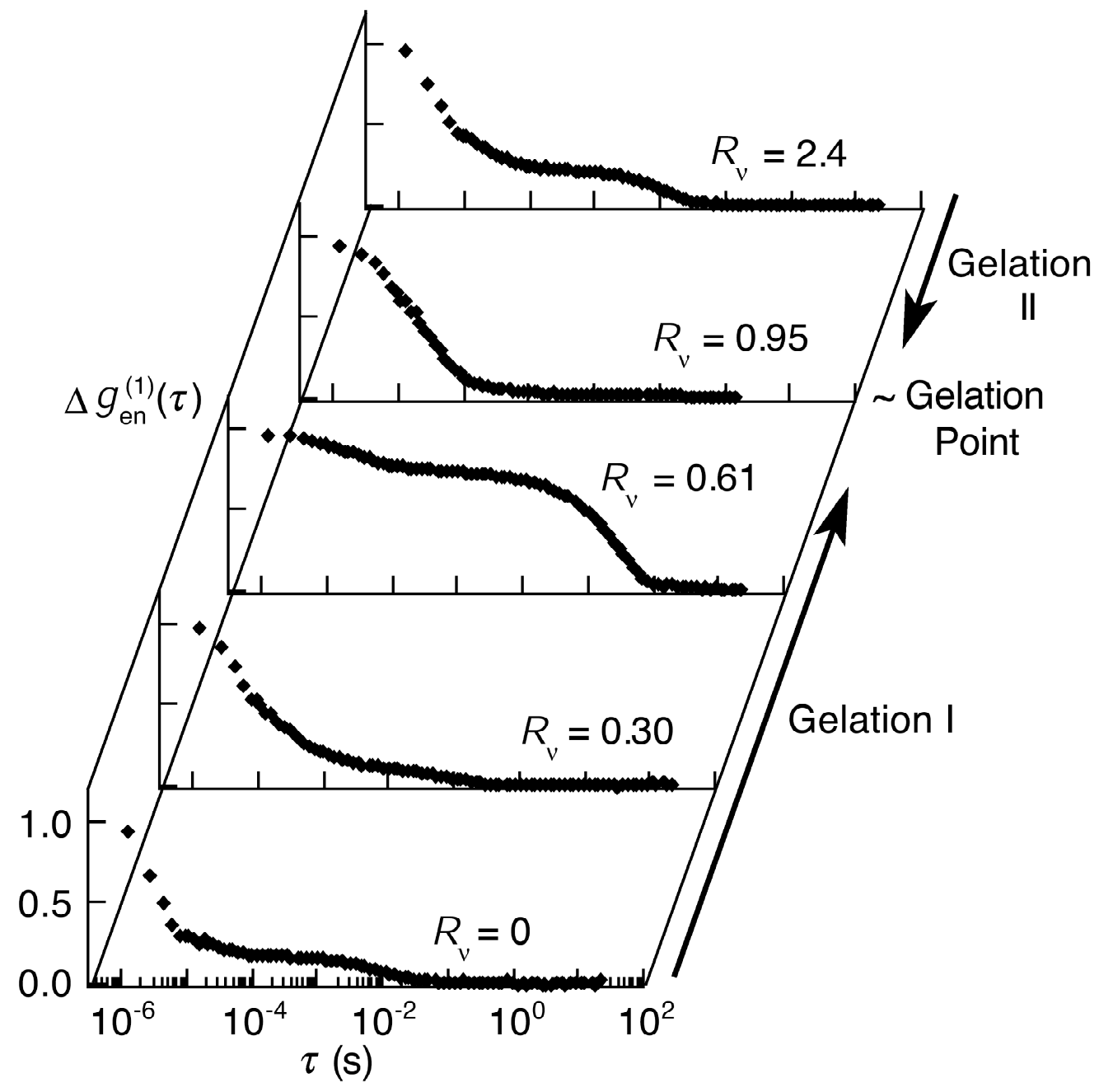

Figure 1. Dynamic component of ensemble-averaged autocorrelation functions $\Delta g_{e n}^{(1)}(\tau)$ at various end-crosslinker concentrations $R_{v}$. Scattering angle is $60^{\circ}$. 


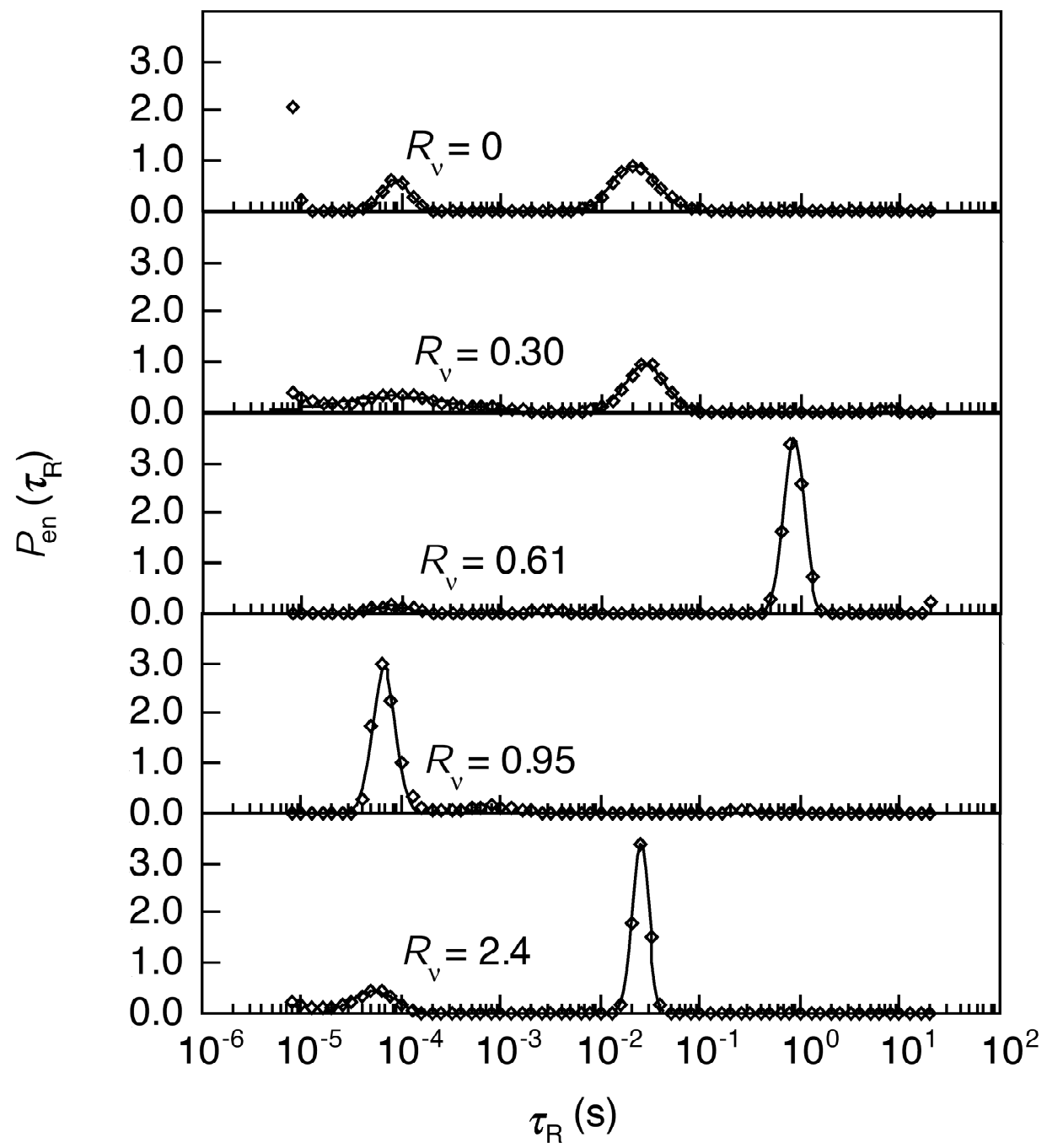

Figure 2. Relaxation-time distribution function $P_{\text {en }}\left(\tau_{R}\right)$ at various end-crosslinker concentration $R_{v}$. Scattering angle is $40^{\circ}$. The solid curves are the logarithmic Gaussian distributions fitted to $P_{\mathrm{en}}\left(\tau_{\mathrm{R}}\right)$. 


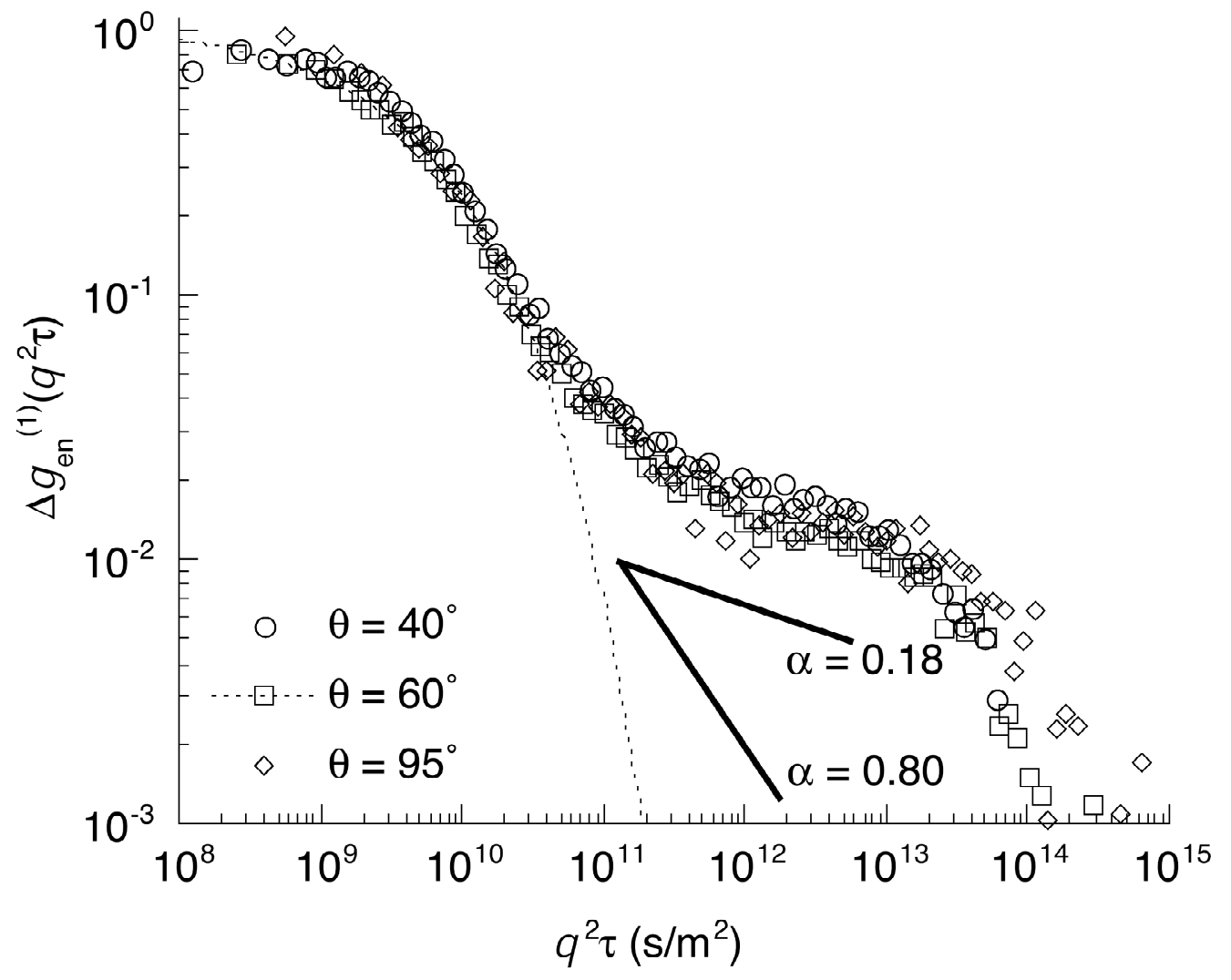

Figure 3. Profiles of $q^{2} \tau$-scaled autocorrelation function $\Delta g_{\mathrm{en}}^{(1)}\left(q^{2} \tau\right)$ near the gelation point at $R_{v}=0.95$. The broken curve is the stretched exponential function fitted to $\Delta g_{\mathrm{en}}^{(1)}\left(q^{2} \tau\right)$ of $\theta=60^{\circ}$. 


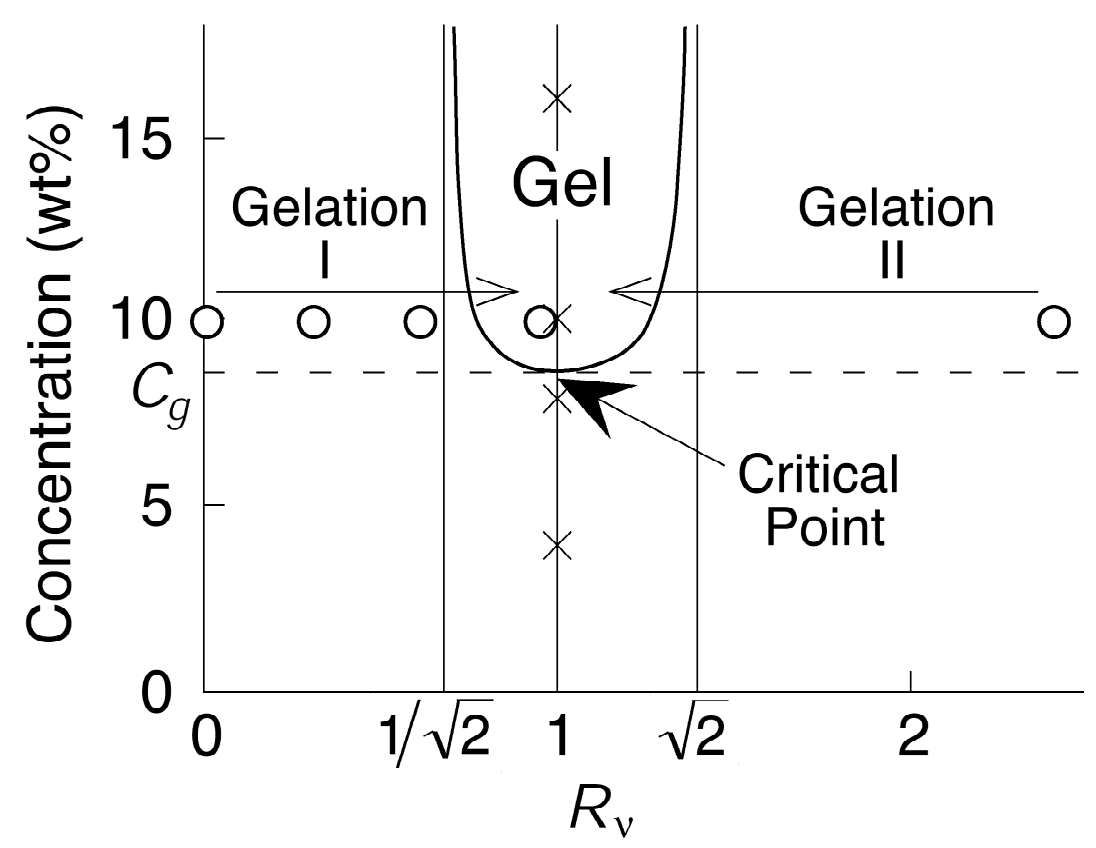

Figure 4. An expected phase diagram of gelation, where the symbols indicate the prepared samples in order to choose a proper polymer concentration for gel formation $(\times)$ and the prepared samples for SMILS measurements $(\bigcirc)$. 